\title{
Choosing Wisely Portugal - Escolhas Criteriosas em Saúde
}

\section{Choosing Wisely Portugal - Wise Health Decisions}

\author{
Miguel BIGOTTE VIEIRA $\rrbracket^{* 1,2,3}$, Guilherme FERREIRA-DOS-SANTOS*2,3,4, Catarina Reis de CARVALHO ${ }^{2,5}$, \\ Catarina Viegas DIAS ${ }^{2,6}$, David Cordeiro SOUSA ${ }^{2,7}$, Inês LEAL ${ }^{2,7}$, João VALENTE JORGE ${ }^{2,8}$, Mariana ALVES ' $^{2,9}$, \\ Mariana MORGADO ${ }^{2,10}$, Rute Baeta BAPTISTA ${ }^{2,11}$, Paula FORTUNATO ${ }^{12}$, António VAZ-CARNEIRO ${ }^{2,3}$, \\ Miguel GUIMARÃES 12,13 \\ Acta Med Port 2018 Oct;31(10):521-523 - https://doi.org/10.20344/amp.11138
}

Palavras-chave: Adesão a Directrizes; Medicina Baseada em Evidências; Melhoria de Qualidade; Padrões de Prática de Médica; Portugal; Tomada de Decisões

Keywords: Decision Making; Evidence-Based Medicine; Guideline Adherence; Portugal; Practice Patterns, Physicians; Quality Improvement

Choosing Wisely Portugal - Escolhas Criteriosas em Saúde é um programa inovador de educação para a saúde. O programa Choosing Wisely foi fundado em 2012 pelo American Board of Internal Medicine e foi posteriormente adaptado e implementado em mais de 20 países, incluindo Alemanha, Austrália, Canadá, Dinamarca, Holanda, Itália, Japão, Nova Zelândia, Reino Unido e Suíça. ${ }^{1,2}$ O objetivo inicial do programa foi fomentar a discussão sobre os procedimentos diagnósticos e terapêuticos que não apresentam benefícios para os doentes e que podem estar associados a riscos desnecessários. Num estudo realizado em 2013 que inquiriu 1000 portugueses, cerca de $99 \%$ considerava que deveria realizar análises de rotina anualmente e $70 \%$ que deveria realizar radiografias de tórax a cada 15 meses. ${ }^{3}$ Um estudo posterior do mesmo grupo avaliou a importância atribuída pelos doentes a várias medidas preventivas e concluiu que os doentes tendem a sobrestimar a importância de medidas preventivas, a atribuir maior importância à realização de exames do que a alterações de estilo de vida, a não identificar quais os exames cuja realização se baseia em evidência e a desconhecer o conceito de individualização de risco. Estas convicções conduzem à solicitação frequente destas intervenções ao profissional de saúde. ${ }^{4} \mathrm{~A}$ implementação deste programa em Portugal pretende promover escolhas criteriosas em saúde e facilitar a comunicação entre profissionais de saúde e doentes.

O desenvolvimento tecnológico promoveu o aumento do número de intervenções diagnósticas e terapêuticas disponíveis em cada situação clínica. Existem vários fatores que podem contribuir para a prescrição de intervenções sem benefício e com potenciais riscos para o doente: i) o desconhecimento da evidência científica existente; ii) o otimismo sobre o seu potencial benefício; iii) a aversão ao risco e consequente prática de medicina defensiva; iv) a ausência de coordenação entre várias especialidades médicas; v) a existência de pressões de índole comercial ou política; vi) a convicção de que um número maior de intervenções corresponde a melhores resultados em saúde e vii) a pressão dos doentes para que a intervenção seja prescrita. ${ }^{2}$ Exemplos de potenciais riscos associados a estas intervenções incluem a ansiedade gerada por resultados falsos positivos, a exposição a radiação emitida por meios complementares de diagnóstico e os efeitos adversos de determinadas intervenções terapêuticas, para além de contribuírem para a ineficiência do sistema de saúde.

A implementação prévia deste programa em diversos países permite-nos aprender com a experiência entretanto adquirida, existindo cinco princípios fundamentais a ser considerados. O programa deverá ser: 1) liderado por médicos; 2) centrado no doente; 3) baseado na evidência científica disponível à data da elaboração de cada recomendação; 4) multidisciplinar; 5) transparente. ${ }^{1}$ Entendemos que o programa deve ser promovido por uma entidade cientificamente competente, idónea e independente das envolvidas no financiamento dos serviços de saúde - em Portugal,

\footnotetext{
* Co-primeiros autores.

1. Serviço de Nefrologia e Transplantação Renal. Centro Hospitalar Lisboa Norte. Lisboa. Portugal.

2. Centro de Estudos de Medicina Baseada na Evidência. Faculdade de Medicina. Universidade de Lisboa. Centro Académico de Medicina de Lisboa. Lisboa. Portugal.

3. Cochrane Portugal. Lisboa. Portugal.

4. Departamento de Medicina Física e Reabilitação. Centro Hospitalar de Lisboa Central. Lisboa. Portugal.

5. Departamento de Obstetrícia. Ginecologia e Medicina da Reprodução. Centro Hospitalar Lisboa Norte. Lisboa. Portugal.

6. NOVA Medical School. Lisboa. Portugal.

7. Serviço de Oftalmologia. Centro Hospitalar Lisboa Norte. Lisboa. Portugal.

8. Serviço de Anestesiologia. Centro Hospitalar Lisboa Norte. Lisboa. Portugal.

9. Serviço de Medicina III. Centro Hospitalar Lisboa Norte. Lisboa. Portugal.

10. Serviço de Cirurgia Pediátrica. Centro Hospitalar Lisboa Norte. Lisboa. Portugal.

11. Serviço de Pediatria Médica. Área da Mulher, Criança e Adolescente. Hospital de Dona Estefânia. Centro Hospitalar Lisboa Central. Lisboa. Portugal.

12. Ordem dos Médicos. Lisboa. Portugal.

13. Serviço de Urologia. Centro Hospitalar de São João. Porto. Portugal.

$\triangle$ Autor correspondente: Miguel Bigotte Vieira. mbigottevieira@gmail.com

Recebido: 02 de agosto de 2018 - Aceite: 20 de agosto de 2018 | Copyright $\odot$ Ordem dos Médicos 2018
} 
a Ordem dos Médicos. Este é um programa centrado no doente, que tem como objetivos a melhoria da qualidade dos serviços de saúde prestados, a prevenção da ocorrência de dano e a promoção da discussão sobre qual a intervenção mais adequada em cada situação clínica. Pretende ainda otimizar a comunicação entre doentes e profissionais de saúde, facilitando a tomada de decisões informadas, individualizadas e partilhadas em saúde.

As recomendações do programa Choosing Wisely Portugal - Escolhas Criteriosas em Saúde incidirão preferencialmente sobre procedimentos frequentes na prática clínica, serão elaboradas por médicos peritos e emitidas pelos Colégios da Especialidade da Ordem dos Médicos. As recomendações emitidas por determinado Colégio da Especialidade poderão ser subscritas por outros Colégios da Especialidade. Poderão ser emitidas recomendações originais ou serem traduzidas e adaptadas à realidade portuguesa recomendações de programas Choosing Wisely internacionais. As recomendações serão baseadas na meIhor evidência científica disponível à data da sua elaboração, redigidas em linguagem acessível, identificadas com os logótipos do programa (Fig.s 1 e 2) e estarão disponíveis para consulta e download nas redes sociais e na página do programa, o qual estará integrado no site da Ordem dos Médicos. Outras entidades da área da saúde poderão apoiar e divulgar o programa, incluindo sociedades científicas e associações de doentes. A gestão deste programa será assegurada por uma equipa multidisciplinar constituída pelos autores do presente artigo.

Este projeto poderá ter diversas aplicações. No estrangeiro, as recomendações foram previamente incorporadas em registos médicos eletrónicos, sendo emitido um alerta com o texto da recomendação sempre que o profissional de saúde solicite intervenções mencionadas nas recomendações. Através desta medida verificou-se ter ocorrido uma diminuição significativa na prescrição de intervenções sem benefício e/ou com riscos associados para os doentes. ${ }^{5}$
Os materiais pedagógicos disponibilizados neste programa poderão ser utilizados em sessões de formação destinadas a doentes e/ou profissionais de saúde, integrados nos currículos dos cursos da área da saúde ou apresentados a doentes durante a consulta. Estes serão particularmente úteis em situações nas quais os doentes manifestem insistentemente interesse na prescrição de meios complementares de diagnóstico e terapêutica sem justificação clínica e com potenciais riscos para a sua saúde. Permitirão ainda que os doentes consultem autonomamente informação de saúde proveniente de uma fonte fidedigna e validada por médicos.

O programa Choosing Wisely Portugal - Escolhas Criteriosas em Saúde foi lançado em Portugal em outubro de 2018. As primeiras recomendações encontram-se já disponíveis (https://ordemdosmedicos.pt/escolhas-criteriosas-em-saude), prevendo-se a inclusão de novas recomendações nos próximos meses. Entusiasma-nos a disponibilidade para colaborar manifestada pela generalidade dos Colégios da Especialidade da Ordem dos Médicos. Apesar de estarmos cientes que a implementação deste programa será morosa, implicará esforço e dedicação de todos os intervenientes, consideramos que os seus objetivos e potenciais ganhos em saúde o justificam. Um dos principais receios dos médicos sobre o programa é a convicção de que os doentes se mostrarão renitentes em aceitar a prescrição de um menor número de procedimentos diagnósticos e/ou terapêuticos. No entanto, verificou-se que doentes mais informados optam frequentemente por realizar menos intervenções. ${ }^{2}$ Consideramos que o sucesso deste programa dependerá da colaboração dos Colégios da Especialidade da Ordem dos Médicos e de todos os médicos, em particular na utilização frequente das recomendações e na sua divulgação junto de doentes e de outros profissionais de saúde. São ainda desejáveis eventuais sugestões, as quais poderão ser enviadas para o seguinte endereço de e-mail: cwportugal@ordemdosmedicos.pt

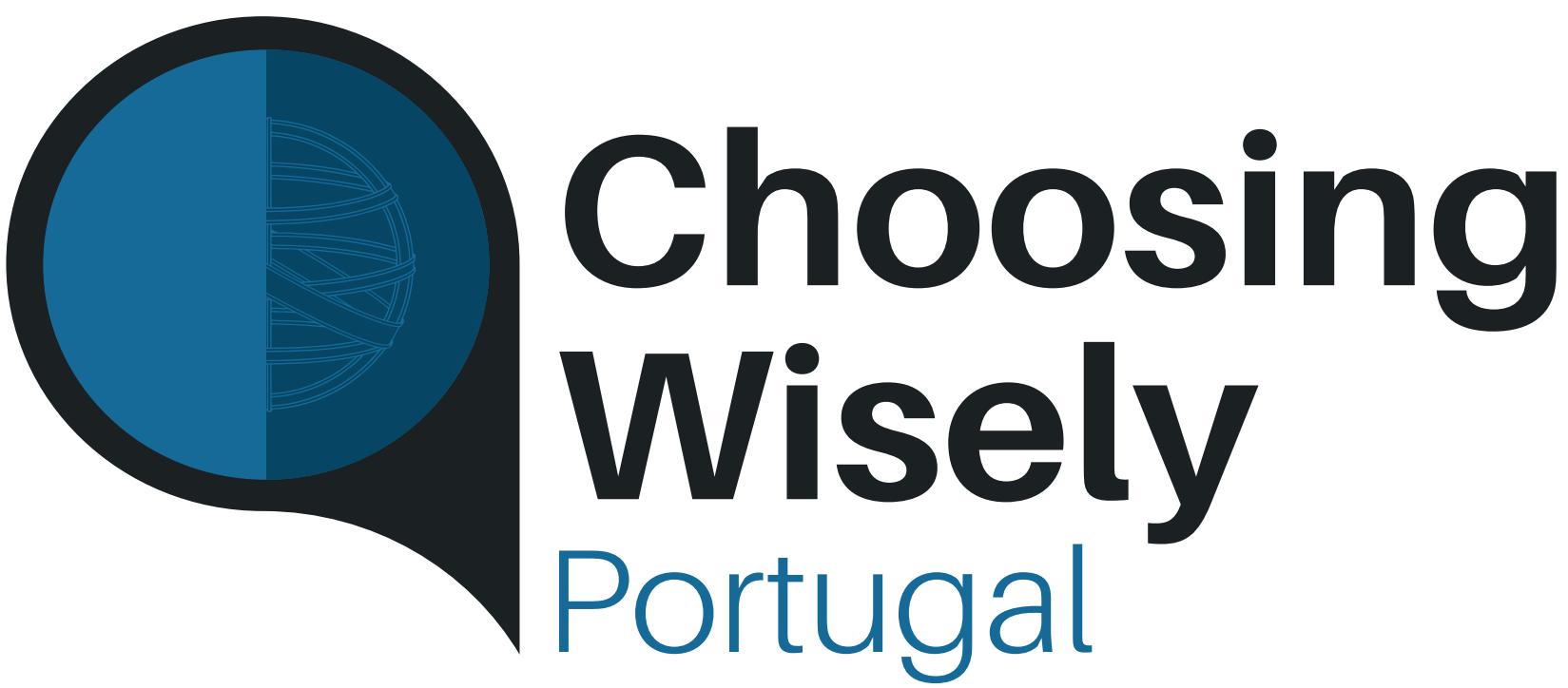

Figura 1 - Logotipo principal do programa Choosing Wisely Portugal - Escolhas Criteriosas em Saúde 


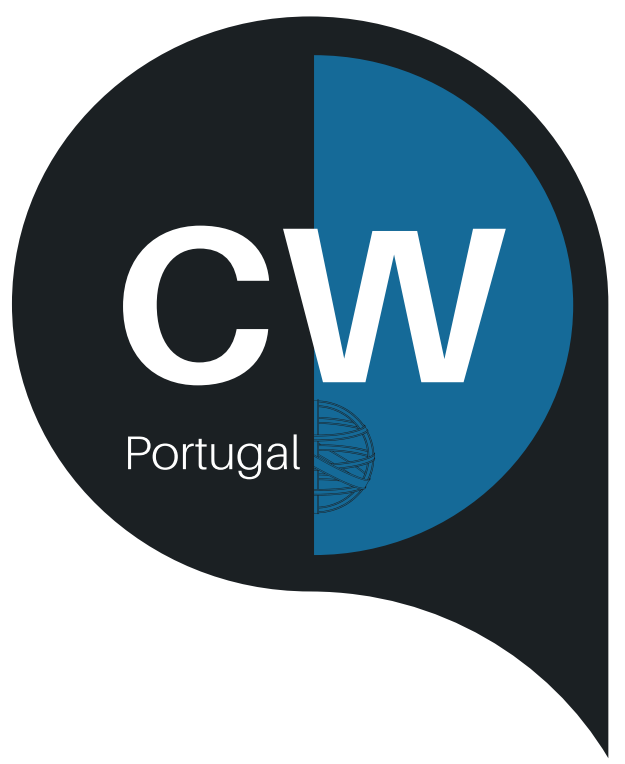

Figura 2 - Logotipo secundário do programa Choosing Wisely Portugal - Escolhas Criteriosas em Saúde

Em conclusão, o programa Choosing Wisely Portugal Escolhas Criteriosas em Saúde pretende reduzir a realização de intervenções com relação risco/benefício desfavorável, melhorar a qualidade dos serviços de saúde prestados à população portuguesa e a comunicação entre doentes e profissionais de saúde. Ao implementar este programa a Ordem dos Médicos desempenha, uma vez mais, o seu papel de provedora dos doentes, pugnando pela qualidade da Medicina praticada em Portugal.

\section{REFERENCIAS}

1. Levinson W, Kallewaard M, Bhatia RS, Wolfson D, Shortt S, Kerr EA, et al. "Choosing Wisely": a growing international campaign. BMJ Qual Saf. 2015;24:167-74.

2. Ross J, Santhirapala R, MacEwen C, Coulter A. Helping patients choose wisely. BMJ. 2018;361:k2585.

3. Martins C, Azevedo LF, Ribeiro O, Sá L, Santos P, Couto L, et al. A population-based nationwide cross-sectional study on preventive health services utilization in Portugal--what services (and frequencies) are

\section{AGRADECIMENTOS}

Os autores agradecem aos Colégios da Especialidade da Ordem dos Médicos a elaboração das recomendações deste programa.

\section{CONFLITOS DE INTERESSE}

Os autores declaram não ter qualquer conflito de interesse relativamente ao presente artigo.

deemed necessary by patients? PLoS One. 2013;8:e81256.

4. Sá L, Ribeiro O, Azevedo LF, Couto L, Costa-Pereira A, Hespanhol A, et al. Patients' estimations of the importance of preventive health services: a nationwide, population-based cross-sectional study in Portugal. BMJ Open. 2016:6:e011755.

5. Lenzer J. Choosing Wisely: setbacks and progress. BMJ. 2015;351:h6760. 